\title{
Protocolo das possibilidades técnicas de aplicação dos sistemas adesivos universais: revisão de literatura com relato de caso
}

\author{
Clinical strategies using universal adhesive systems: literature review with case report
}

\section{Letícia de Souza Lopes}

Departamento de Formação Específica em Odontologia, Faculdade de Odontologia, Universidade Federal Fluminense, Nova Friburgo, Rio de Janeiro, Brasil

Pâmela Malaquias

Pós-graduação em Odontologia, Universidade Estadual de Ponta Grossa, Ponta Grossa, Paraná, Brasil

Fernanda Signorelli Calazans

Departamento de Formação Específica em Odontologia, Faculdade de Odontologia, Universidade Federal Fluminense, Nova Friburgo, Rio de Janeiro, Brasil

Alessandra Reis

Pós-graduação em Odontologia, Universidade Estadual de Ponta Grossa, Ponta Grossa, Paraná, Brasil

Alessandro Dourado Loguércio

Pós-graduação em Odontologia, Universidade Estadual de Ponta Grossa, Ponta Grossa, Paraná, Brasil

Marcos de Oliveira Barceleiro

Departamento de Formação Específica em Odontologia, Faculdade de Odontologia, Universidade Federal Fluminense, Nova Friburgo, Rio de Janeiro, Brasil

\section{ResUmo}

Este relato de caso demonstra a utilização clínica de um novo sistema adesivo universal, Xeno Universal (Dentsply DeTrey, Alemanha) descrevendo quatro formas possíveis de aplicação: autocondicionante, com ou sem condicionamento seletivo do esmalte, ou com condicionamento ácido total, seguido da aplicação do adesivo sobre dentina seca ou úmida. Os adesivos universais de uma maneira geral são de fácil aplicação e versáteis, uma vez que o mesmo produto pode ter várias formas de aplicação, reduzindo o tempo clínico e em muitas situações a sensibilidade técnica. No entanto, mais estudos laboratoriais e clínicos que comprovem a eficácia deste novo adesivo universal em particular são necessários, antes que seu uso seja bem indicado aos cirurgiões-dentistas.

Palavras-chave: adesivos dentinários; condicionamento ácido dentário.

\section{Abstract}

This case report describes the clinical application of a new universal adhesive system, Xeno Universal (Dentsply DeTrey, Germany) describing four application modes: self-etching, with or without selective enamel etching, or total etching followed by adhesive application on dry or moist dentin. Universal adhesive systems usually are versatile and easy to use, facilitating the operator, since the same product has several technical possibilities, thus reducing the operating time and the technique sensitivity. However, long-term laboratory studies and more clinical reports and/or clinical trials are needed to prove the quality of this new universal adhesive system, before dentists can start using them in their dental offices.

Keywords: dentin-bonding agents; acid etching dental.

\section{Introdução}

$\mathbf{0}$

s sistemas adesivos revolucionaram a Odontologia estética, proporcionando ao cirurgião-dentista a possibilidade de oferecer restaurações com melhor qualidade estética, sendo feita de maneira direta, em consulta única e com propriedades mecânicas satisfatórias. ${ }^{1}$

Os sistemas adesivos convencionais dependem da desmineralização de esmalte e dentina, realizada tradicionalmente por um ácido fosfórico, para posterior hibridização do substrato dentário. ${ }^{1} \mathrm{Na}$ maioria dos sistemas adesivos, a profundidade do padrão de condicionamento desempenha um papel significativo na magnitude da força de adesão ao esmalte. ${ }^{2} \mathrm{E}$ na dentina, a remoção da lama dentinária, seguida da desmineralização da dentina e a consequente exposição das fibras colágenas é essencial para que ocorra a posterior hibridização. ${ }^{3}$ Embora estes sistemas adesivos já tenham sido extensamente avaliados, in vitro e in vivo, normalmente com excelentes resultados, ${ }^{4}$ eles se mostram extremamente sensíveis à técnica, $\mathrm{e}$ alguns clínicos os consideram confusos e complexos de serem utilizados, fazendo com que estes não sejam explorados da melhor maneira possível, causando assim um série de problemas, dentre os quais, os mais comuns são a sensibilidade pós-operatória, manchamento marginal e falhas adesivas após algum tempo em uso na cavidade oral. ${ }^{5}$

Os sistemas adesivos autocondicionantes surgiram no mercado com uma nova proposta de adesão, que teoricamente permitiria uma simplificação da técnica. Estes sistemas adesivos autocondicionantes dependem da desmineralização do tecido dentário por um primer acídico associado ao adesivo, que permitiria uma desmineralização e simultânea infiltração no tecido dentinário, o que formaria assim, de maneira imediata, uma hibridização teoricamente com um percentual menor de falhas, e que consequentemente levaria a menos problemas clínicos, como os descritos anteriormente. Resultados in vitro e in vivo têm demonstrado de uma maneira geral que estes sistemas adesivos autocondicionantes se mostram bastante eficazes em dentina, no entanto, os resultados em esmalte normalmente se apresentam desfavoráveis. ${ }^{6}$ Desta maneira, a técnica de condicionamento ácido seletivo do esmalte surgiu para tentar melhorar a adesão a este substrato. Nesta técnica, o cirurgião-dentista realiza o condicionamento do esmalte de maneira tradicional, e, em seguida, faz a aplicação de um sistema adesivo 
autocondicionante, tendo assim as vantagens da adesão tradicional no esmalte e as vantagens da adesão com adesivos autocondicionantes na dentina.

Alguns estudos mostraram, no entanto que, com o uso do condicionamento ácido seletivo do esmalte, havia o risco de haver contaminação da dentina com o ácido fosfórico e, neste caso, o adesivo autocondicionante seria utilizado em dentina já condicionada, o que diminuiria a qualidade da hibridização. ${ }^{7}$ Assim, procurando solucionar estes problemas, surgiram os novos sistemas adesivos chamados Universais, que poderiam teoricamente ser utilizados com ou sem o condicionamento, tanto em esmalte quanto em dentina.

Estes sistemas adesivos universais são em sua essência autocondicionantes. Os fabricantes desta nova modalidade relatam que estes sistemas podem ser utilizados com ou sem condicionamento do esmalte e da dentina. Sabe-se, no entanto, que o condicionamento com ácido fosfórico aumenta significativamente a resistência de união dos adesivos autocondicionantes ao esmalte ${ }^{8} \mathrm{e}$, por isso, o condicionamento seletivo de margens de esmalte também tem sido recomendado por alguns autores, antes da aplicação destes novos sistemas adesivos autocondicionantes universais. ${ }^{8}$

Diferentes centros de pesquisa já mostraram que estes adesivos universais demonstraram bons resultados de resistência de união em dentina condicionada ou não, ${ }^{9} \mathrm{e}$ isto tem sido atribuído aos monômeros acídicos incorporados na formulação química destes adesivos, que seriam capazes de promover união química ao dente. ${ }^{10}$ Assim, considerando a maior simplificação da etapa de aplicação dos sistemas adesivos, quando da utilização destes sistemas adesivos universais, este relato de caso se propõe a demonstrar todas as possibilidades de utilização clínica de um novo sistema adesivo universal (autocondicionante com e sem condicionamento seletivo em esmalte, condicionamento ácido total e aplicação do sistema adesivo em dentina seca ou em dentina úmida), com a posterior discussão sobre o potencial de utilização dos mesmos na prática clínica diária.

\section{Relato de Caso}

Paciente do sexo masculino, 45 anos de idade, apresentou no exame clínico lesões cervicais não cariosas (LCNC) nos dentes 32, 31, 41 e 42 , todas com características semelhantes: ausência de sensibilidade ao ar, à sondagem ou espontânea, grau de esclerose tipo 1 (ausência de esclerose, a dentina encontrava-se ligeiramente amarelada, com pouca descoloração), $50 \%$ da margem em esmalte e $50 \%$ em dentina, profundidade de, aproximadamente, $1 \mathrm{~mm}$ e angulação de $90^{\circ}$ e sem facetas oclusais de desgaste (Figura 1). Todas as cavidades mediam $4 \mathrm{~mm}$ no sentido mésio-distal e apresentavam as seguintes medidas no sentido cérvico-incisal: dente 32 e dente $41-2 \mathrm{~mm}$; dente $31-3 \mathrm{~mm}$; dente $42-1 \mathrm{~mm}$. O paciente recebeu instrução de higiene oral pelos operadores. Também foi realizada profilaxia com pedra-pomes e água com taça de borracha, em contra-ângulo de baixa velocidade (Gnatus, Ribeirão Preto, SP).

Antes de realizar o isolamento absoluto da cavidade com lençol de borracha (Madeitex, São José dos Campos, SP) e grampo 212 modificado (SS White Duflex, Rio de Janeiro, RJ), o paciente foi anestesiado com mepivacaína 3\% (Mepisy, Nova DFL, Rio de Janeiro, RJ, Brasil) e todas as cavidades a serem restauradas receberam profilaxia com pasta de pedra-pomes e água. A seleção de cor feita com uso da escala Vita Classical também foi realizada antes da realização do isolamento absoluto. Seguindo as orientações da ADA, ${ }^{11}$ os operadores não prepararam qualquer retenção adicional ou bisel.

As LCNC receberam o sistema adesivo Xeno Select (Dentsply DeTrey, Konstanz, Alemanha) aplicado de diferentes modos, de acordo com os diferentes grupos, sendo três técnicas indicadas pelo fabricante, e uma testada pelos autores deste relato:

\section{1 - Condicionamento Ácido}

Condicionamento total mantendo a dentina seca (técnica não descrita pelo fabricante deste sistema adesivo): foi feita a aplicação do ácido fosfórico à 37\% (Dentsply, Petrópolis, RJ, Brasil) através da seringa do produto, primeiramente em esmalte por 15 segundos, estendendo para a dentina por mais 15 segundos (Figura 2a). Após a aplicação por 30 segundos em esmalte e $15 \mathrm{em}$ dentina foi feita a lavagem por 60 segundos e em seguida a secagem com jato de ar indireto por 10 segundos, mantendo a cavidade em dentina com aspecto seco e sem brilho (Figura 3a).

Condicionamento total mantendo a dentina úmida: foi feita a aplicação do ácido fosfórico à 37\% (Dentsply, Brasil) através da seringa do produto, primeiramente em esmalte por 15 segundos, estendendo para a dentina por mais 15 segundos. Após a aplicação por 30 segundos em esmalte e 15 em dentina foi feita a lavagem por 60 segundos e em seguida a secagem com jato de ar indireto por 5 segundos, mantendo a cavidade em dentina com aspecto brilhoso (Figura $3 b$ ).

Condicionamento seletivo de esmalte: foi feita aplicação de ácido fosfórico à 37\% (Dentsply, Brasil) através da seringa do produto, apenas em esmalte (Figura 2b). Após 30 segundos foi feita a lavagem abundante com água por 60 segundos e em seguida a secagem até a total evaporação da água da superfície da cavidade.

Autocondicionante: $\mathrm{O}$ sistema adesivo foi aplicado na superfície seca da cavidade sem qualquer condicionamento, seguido do protocolo restaurador, conforme descrição a seguir.

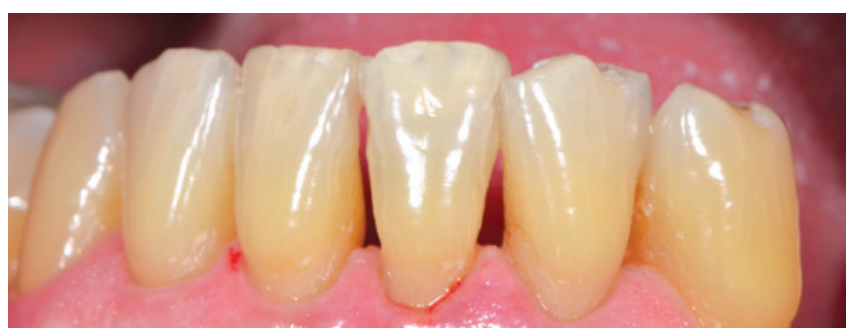

Figura 1. Aspecto inicial das lesões cervicais não cariosas. Observar grau de esclerose grau 1, margens das cavidades e profundidade das mesmas 

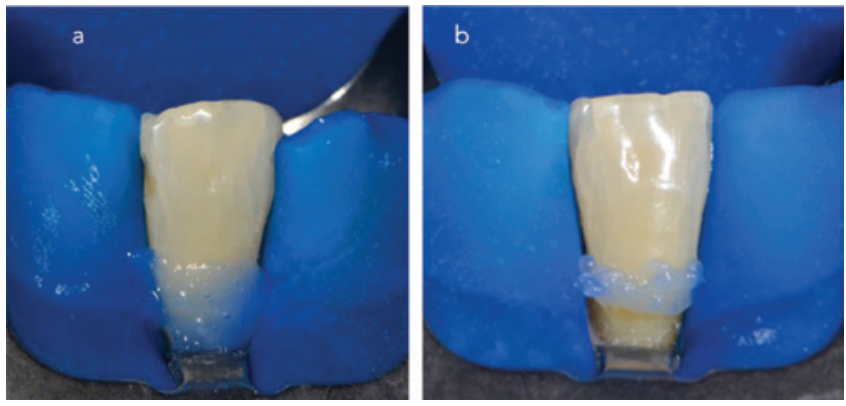

Figura 2. Etapa de condicionamento ácido do dente. (a) condicionamento no esmalte e dentina; (b) condicionamento seletivo do esmalte

\section{2 - Aplicação do Sistema Adesivo}

Nas quatro modalidades de aplicação descritas no item anterior, com ou sem condicionamento ácido, o sistema adesivo foi aplicado de maneira ativa (o pincel embebido no sistema adesivo foi esfregado durante todo o tempo) sobre toda superfície da cavidade por 20 segundos, de acordo com as instruções do fabricante. O pincel descartável "Flocker Applicator Tips" (Dentsply, Alemanha) foi agitado sobre a superfície da cavidade sob pressão manual. Em seguida, para completa evaporação do solvente, aplicou-se um jato moderado de ar limpo e seco de forma próxima e suficientemente forte para manter a camada de adesivo em movimento ligeiro. Esta evaporação do solvente continuou com mais ar forçado até não haver movimentos no adesivo, por pelo menos 5 segundos. Neste momento, a superfície deveria apresentar um aspecto brilhante e uniforme, sem aparência de poças (excesso de sistema adesivo). Finalmente, o sistema adesivo foi fotoativado por 10 segundos com fotopolimerizador com potência de $1200 \mathrm{~mW} / \mathrm{cm}^{2}$ (Radii Cal, SDI, Victoria, Australia). O fotopolimerizador foi posicionado justaposto à cavidade.

\section{3 - Restauração}

Após a aplicação do sistema adesivo, as cavidades foram restauradas com a resina composta EvoluX (Dentsply, Brasil), aplicada em até 3 incrementos, cada um sendo fotoativado por 30 segundos com o mesmo fotopolimerizador utilizado anteriormente nos sistemas adesivos, também posicionado justaposto ao material restaurador. Todas as restaurações foram iniciadas sempre pelo incremento mais cervical (Figura 4a). As restaurações receberam acabamento com pontas diamantadas 2200FF (KG Sorensen, Barueri, SP, Brasil) em alta rotação, com irrigação constante. O polimento foi realizado com pontas de borracha Enhance e PoGo (Dentsply, Brasil), uma semana após a colocação das restaurações (Figura 4b). Este paciente encontrava-se em acompanhamento de 6 meses no momento da confecção deste artigo e faz parte de um projeto maior de avaliação do sistema adesivo ora utilizado.
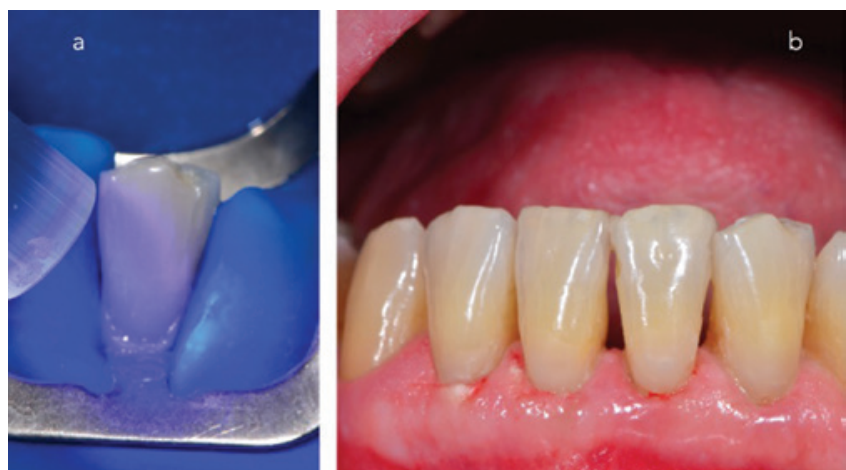

Figura 4. Etapa de restauração com a resina composta, de maneira similar nos quatro dentes restaurados. (a) início pelo incremento mais cervical; (b) aspecto final imediato das restaurações

\section{Discussão}

As LCNC devem ser restauradas da maneira mais conservadora possível, portanto, materiais adesivos são os mais indicados, ${ }^{12}$ sendo as mais utilizadas as restaurações em que sistemas adesivos e resinas compostas são empregados. Os sistemas adesivos são classificados com relação ao seu mecanismo de ação. São eles: convencionais, os quais exigem uma etapa de condicionamento ácido prévio com lavagem do mesmo e os autocondicionantes, os quais possuem um primer acídico em sua composição, não sendo necessária a etapa de ácido prévio e lavagem. ${ }^{13}$

A tecnologia evolui rápida e continuamente e, como não poderia ser diferente, novos sistemas adesivos estão disponíveis no mercado odontológico. Por isso, o objetivo desse relato de caso foi demonstrar o protocolo de utilização de um sistema adesivo universal e suas quatro possibilidades técnicas. Com essas possibilidades técnicas, pode-se aliar a eficácia dos sistemas adesivos convencionais em esmalte e a dos adesivos autocondicionantes em dentina, diminuindo a sensibilidade técnica e facilitando o operador.

Os sistemas universais têm uma vantagem com relação ao tempo e a facilidade de trabalho, já que em um único frasco 
estão presentes o primer acídico e o adesivo propriamente dito. Portanto, a técnica de aplicação torna-se rápida em relação aos outros tipos de sistemas adesivos e também muito mais fácil, principalmente quando se utiliza a técnica autocondicionante e de condicionamento seletivo de esmalte. No caso do sistema adesivo utilizado neste relato, particularmente, os operadores encontraram facilidade em seu uso devido a uma viscosidade ideal, já que o adesivo não era tão fluido a ponto de cair facilmente do frasco ou do aplicador descartável, nem tão viscoso, o que poderia dificultar sua aplicação, mesmo que vigorosa.

A forma de aplicação do material pode explicar os resultados clínicos, em especial, a aplicação vigorosa (ativa). Já foi demonstrado que a aplicação vigorosa de adesivos autocondicionantes melhora a resistência de união imediata e diminui a degradação da união em longo prazo. ${ }^{14,15} \mathrm{Da}$ mesma forma, a aplicação vigorosa melhora a adesão imediata de adesivos convencionais, mesmo quando aplicados em dentina seca e esta adesão foi mantida ao longo do tempo em estudos laboratoriais e clínicos. Isto ocorre porque a aplicação vigorosa melhora a infiltração dos monômeros na dentina, sejam eles adesivos convencionais ou autocondicionantes, e colabora na evaporação do solvente. ${ }^{16,17}$

No caso de Xeno Select, este novo sistema adesivo contém um solvente tert-butanol especial (2-metil-2-propanol), que de acordo com o fabricante é capaz de reexpandir fibrilas de colagéno colapsados. Durante a análise microscópica das interfaces resina-dentina produzidas por um sistema adesivo (XP-Bond) contendo tert-butanol, foi observada a formação de camadas híbridas muito similares tanto em dentina seca quanto em dentina úmida. ${ }^{18}$ Da mesma forma, embora o manual de instruções de utilização deste sistema adesivo não contemple a técnica de condicionamento ácido total e posterior secagem da dentina, deixando-a seca, descrita neste relato, o compêndio científico ${ }^{19}$ deste mesmo produto, editado pelo fabricante do mesmo, cita em todos os momentos a técnica com condicionamento e posterior secagem da dentina, deixando-a seca. No referido compêndio, o sistema Xeno Select foi comparado com o seu antecessor Xeno V e também com o Sistema adesivo Single Bond Universal (3M ESPE), em testes de resistência ao cisalhamento em esmalte e dentina, e também foi feita análise microscópica da camada híbrida. Em todos estes testes, o sistema adesivo foi utilizado também na técnica descrita neste relato, com condicionamento total e posterior secagem da dentina, até que a mesma ficasse seca. Por isso, neste relato, houve a descrição de utilização do sistema adesivo com quatro técnicas, sendo três descritas no manual de instruções, e mais uma com aplicação em dentina seca.

Quando se aplica o adesivo com o condicionamento ácido da dentina, as microporosidades advindas deste procedimento garantem o padrão de adesão e a este devem ser atribuídos os bons resultados quando os adesivos, tais como o Xeno Select, são usados na dentina condicionada. Contudo, quando aplicado no modo autocondicionante, é fundamental a presença de monômeros que façam alguma interação química com a dentina. ${ }^{20}$

De acordo com o fabricante do Xeno Select, dois monômeros são responsáveis pela interação com a superfície de dentina: um "inverso" de éster de ácido fosfórico funcionalizado e um ácido alquilsulfônico acriloilamino, que já se encontravam presentes no seu antecessor Xeno $\mathrm{V}^{21}{ }^{21}$ Estes monômeros funcionais inicialmente aderem ao cálcio disponível na superfície dos dentes, ${ }^{22,23}$ porém esta capacidade de união parece não ser muito estável ${ }^{23} \mathrm{o}$ que parece ser indício de que este material não deverá ter uma adesão durável ao dente quando aplicado como autocondicionante. O mesmo grupo de pesquisa envolvido neste caso clínico esta realizando uma avaliação clínica com este produto utilizado nas técnicas aqui descritas. Foram realizadas 124 restaurações e após 6 meses de avaliação, 11 restaurações foram perdidas nos grupos autocondicionante (com e sem condicionamento seletivo em esmalte) contra apenas 04 dos grupos onde o condicionamento ácido total foi aplicado (dentina seca ou úmida)..$^{24}$ Isto indica que este novo material, apesar de ser indicado como Universal não deveria ser aplicado como autocondicionante, confirmando os resultados anteriores de ZHOU et al. ${ }^{25}$ Outros estudos, principalmente clínicos, deveriam ser realizados para confirmar os resultados encontrados neste estudo clínico mencionado. ${ }^{24}$

Apesar do lançamento de novos sistemas adesivos no mercado ocorrer frequentemente, é de grande importância mostrar ao clínico a sua correta forma de aplicação para garantir a longevidade das restaurações. Porém, infelizmente, de acordo com Perdigão et al. ${ }^{26}$ a demanda de novidades constantes no mercado faz com que novas gerações de materiais sejam lançadas sem a análise criteriosa do mesmo. Idealmente, uma vez testada e comprovada a qualidade in vitro destes novos materiais, um ou mais ensaios clínicos deveriam se seguir imediatamente para avaliar a eficácia clínica do adesivo em questão.

Os sistemas adesivos universais, de uma maneira geral, são produtos versáteis e de fácil utilização, facilitando o operador, já que um mesmo produto/frasco possui diversas possibilidades técnicas, diminuindo assim o tempo operatório e a sensibilidade técnica e assim o clínico pode decidir que técnica poderá usar de acordo com cada caso. No entanto, o sistema adesivo utilizado neste relato carece de mais estudos que comprovem sua efetividade e eficiência, a médio e longo prazos. Novos estudos, princioalmente, clínicos de longevidade, são necessários para que a indicação clínica deste material seja feita com maior suporte científico.

\section{Conclusão}

Embora a ideia dos sistemas adesivos universais de uma maneira geral seja bastante promissora, os autores concluíram baseados na literatura existente que o sistema adesivo Xeno Select, utilizado neste relato, não deve ser utilizado como autocondicionante, dependendo assim de condicionamento ácido prévio em esmalte e dentina. 


\section{Referências ::}

1. Pashley DH, Tay FR. Aggressiveness of contemporary self-etching adhesives. Part II:etching effects on unground enamel. Dental Materials. 2001;17(5):430-44.

2. Zafar MS, Ahmed N. The effects of acid etching time on surface mechanical properties of dental hard tissues. Dental Materials Journal. 2015;34(3):315-320.

3. Salvio LA, Hipolito VD, Martins AL, de Goes MF. Hybridization quality and bond strength of adhesive systems according to interaction with dentin. European Journal of Dentistry. 2013;7(3):315-26.

4. Van Dijken JW. A randomized controlled 5-year prospective study of two HEMA-free adhesives, a 1-step self etching and a 3-step etch-and-rinse, in non-carious cervical lesions. Dental Materials. 2013;29(11):e271-80.

5. Karaman E, Yazici AR, Aksoy B, Karabulut E, Ozgunaltay G, Dayangac B. Effect of operator variability on microleakage with different adhesive systems. European Journal of Dentistry. 2013;7(Suppl 1):S60-S65.

6. Rotta M, Bresciani P, Moura SK, Grande RH, Hilgert LA, Baratieri LN, et al. Effects of phosphoric acid pretreatment and substitution of bonding resin on bonding effectiveness of self-etching systems to enamel. Journal of Adhesive Dentistry. 2007;9(6):537-45.

7. Erickson RL, Barkmeier WW, Latta MA. The role of etching in bonding to enamel: a comparison of self-etching and etch-and-rinse adhesive systems. Dental Materials. 2009;25(11):1459-67.

8. Van Landuyt KL, Peumans M, De Munck J, Lambrechts P, Van Meerbeer B. Extension of a one-step self-etch adhesive into a multi-step adhesive. Dental Materials. 2006;22(6):533-44.

9. Muñoz MA, Luque I, Hass V, Reis A, Loguercio AD, \& Bombarda NH. Immediate bonding properties of universal adhesives to dentine. Journal of Dentistry. 2013;41(5):404-11.

10. Muñoz MA, Luque-Martinez I, Malaquias P, Hass V, Reis A, Campanha $\mathrm{NH}$, et al. In vitro longevity of bonding properties of universal adhesives to dentin. Operative Dentistry. 2015;40(3):282-92.

11. American Dental Association Council on Scientific Affairs. Acceptance Program Guidelines: Dentin and Enamel Adhesive Materials. American Dental Association. Chicago, 2001.

12. Overton JD, LittleStar ML, Starr BC. Class 5 restorations. In: Fundamental of operative dentistry. A contemporary approach. 3rd ed. Chicago: Quintessence Publishing Co. Inc.; 2006. p. 420-36.

13. VanMeerbeek B, Peumans M, Poitevin A, Mine A, VanEnde A, De Munck J. Relationship between bond strength tests and clinical outcome. Dental Materials. 2010;26(e):100-21.
14. do Amaral RC, Stanislawczuk R, Zander-Grande C, Gagler D, Reis A, Loguercio AD. Bond strength and quality of the hybrid layer of one-step self-etch adhesives applied with agitation on dentin. Operative Dentistry. 2010;35(2):211-9.

15. Loguercio AD, Stanislawczuk R, Mena-Serrano A, Reis A. Effect of 3-year water storage on the performance of one-step self-etch adhesives applied actively on dentine. Journal of Dentistry. 2011;39(8):578-87.

16. Reis A, Pellizzaro A, Dal-Bianco K, Gones OM, Patzlaff R, Loguercio AD. Impact of adhesive application to wet and dry dentin on long-term resin-dentin bond strengths. Operative Dentistry. 2007;32(4):380-7.

17. Loguercio AD, Raffo J, Bassani F, Balestrini H, Santo D, do Amaral $\mathrm{RC}$, et al. 24-month clinical evaluation in non-carious cervical lesions of a two-step etch-and-rinse adhesive applied using a rubbing motion. Clinical Oral Investigation. 2011;15(4):589-96.

18. Machado LS, Oliveira FG, Guinossi TA, Sundfeld Neto D, Alexandre RS, Reis AF, et al. Effects of dentin dry/moist condition on the immediate adhesive performance of different solvent-based etch-and-rinse adhesive systems. International Journal of Adhesion \& Adhesives. 2013;43(1):1-6.

19. Dentsply De Trey. Scientific Compendium Xeno Select. 2014. Disponível em http://www.dentsply.de/bausteine.net/f/9373/XenoSelectSC140116.pdf

20. Yoshihara K, Yoshida Y, Nagaoka N, Fukegawa D, Hayakawa S, Mine A, et al. Nano-controlled molecular interaction at adhesive interfaces for hard tissue reconstruction. Acta Biomateralia. 2010;6(9):3573-82.

21. Van Loghum BS. Xeno Select Fact File. Tandartspraktijk. 2014;35(5):50-1.

22. Van Meerbeek B, Yoshihara K, Yoshida Y, Mine A, De Munck J, Van Landuyt KL. State of the art of self-etch adhesives. Dental Materials. 2011;27(1):17-28.

23. Maeda T, Yamaguchi K, Takamizawa T, Rikuta A, Tsubota K, Ando S, et al. $\mathrm{pH}$ changes of self-etching primers mixed with powdered dentine. Journal of Dentistry. 2008;36(8):606-10.

24. Lopes LS, Calazans FS, Hidalgo R, Buitrago LL, Gutierrez F, Reis A, et al. Six-month Follow-up of Cervical Composite Restorations Placed With a New Universal Adhesive System: A Randomized Clinical Trial. Operative Dentistry. 2016;41(3). Epub ahead of print.

25. Zhou L, Wang Y, Yang H, Guo J, Tay FR, Huang C. Effect of chemical interaction on the bonding strengths of self-etching adhesives to deproteinised dentine. Journal of Dentistry. 2015;43(8):973-80.

26. Perdigão J, Kose C, Mega-Serrano AP, de Paula EA, Tay LY, Reis A, et al. A New Universal Simplified Adhesive: 18-Month Clinical Evaluation. Operative Dentistry. 2013;39(2):113-27.

Recebido em: 28/03/2016 / Aprovado em: 23/05/2016

Marcos de Oliveira Barceleiro

Faculdade de Odontologia da Universidade Federal Fluminense - Campus de Nova Friburgo

Rua Dr. Silvio Henrique Braune, 22, Centro

Nova Friburgo/ RJ, Brasil - CEP: 28625-650

E-mail: marcosbarceleiro@gmail.com 